OPEN ACCESS

Edited by:

Reinhild Klein,

University of Tübingen, Germany

Reviewed by: Bettina Hohberger,

University of Erlangen

Nuremberg, Germany

Teresa Tsai,

Ruhr-University Bochum, Germany

*Correspondence:

Franz H. Grus

fgrus@eye-research.org

Specialty section:

This article was submitted to

Multiple Sclerosis and

Neuroimmunology,

a section of the journal

Frontiers in Immunology

Received: 12 October 2020 Accepted: 01 February 2021

Published: 19 February 2021

Citation:

Beutgen VM, Pfeiffer N and Grus FH (2021) Serological Levels of Anti-clathrin Antibodies Are Decreased in Patients With Pseudoexfoliation Glaucoma.

Front. Immunol. 12:616421. doi: 10.3389/fimmu.2021.616421

\section{Serological Levels of Anti-clathrin Antibodies Are Decreased in Patients With Pseudoexfoliation Glaucoma}

\author{
Vanessa M. Beutgen, Norbert Pfeiffer and Franz H. Grus*
}

Experimental and Translational Ophthalmology, Department of Ophthalmology, University Medical Center of the Johannes Gutenberg-University Mainz, Mainz, Germany

Evidence for immunologic contribution to glaucoma pathophysiology is steadily increasing in ophthalmic research. Particularly, an altered abundance of circulating autoantibodies to ocular antigens is frequently observed. Here, we report an analysis of autoantibody abundancies to selected antigens in sera of open-angle glaucoma patients, subdivided into normal-tension glaucoma $(N=31)$, primary open-angle glaucoma $(N=43)$ and pseudoexfoliation glaucoma $(N=45)$, vs. a non-glaucomatous control group ( $N=46)$. Serum samples were analyzed by protein microarray, including 38 antigens. Differences in antibody levels were assessed by ANOVA. Five serological antibodies showed significantly altered levels among the four groups $(P<0.05)$, which can be used to cluster the subjects in groups consisting mainly of PEXG or POAG/NTG samples. Among the altered autoantibodies, anti-Clathrin antibodies were identified as most important subgroup predictors, enhancing prospective glaucoma subtype prediction. As a second aim, we wanted to gain further insights into the characteristics of previously identified glaucoma-related antigens and their role in glaucoma pathogenesis. To this end, we used the bioinformatics toolset of Metascape to construct protein-protein interaction networks and GO enrichment analysis. Glaucoma-related antigens were significantly enriched in 13 biological processes, including mRNA metabolism, protein folding, blood coagulation and apoptosis, proposing a link of glaucoma-associated pathways to changes in the autoantibody repertoire. In conclusion, our study provides new aspects of the involvement of natural autoimmunity in glaucoma pathomechanisms and promotes advanced opportunities toward new diagnostic approaches.

Keywords: autoantibodies, autoimmunity, glaucoma, microarray, bioinformatics, immunoproteomics

\section{INTRODUCTION}

Glaucoma is a group of progressive neurodegenerative diseases of the optic nerve with variating forms of manifestation. An open-angle glaucoma (OAG) manifests as atrophy of the optic nerve and resulting vision loss while a normal iridiocorneal angle is maintained. The disease subtypes are assumed to have multifactorial pathogenesis, but few factors can be considered as significant hallmarks. Primary open-angle glaucoma (POAG), the most common form, is characterized by a typical optic nerve cupping, caused by the death of retinal ganglion cells (RGCs) and their axons, along with an increased intraocular pressure (IOP) that is mainly caused by dysregulations in the trabecular meshwork (TM) (1-3). An elevated IOP can also be observed in secondary 
glaucoma forms. In pseudoexfoliation syndrome (PEX), affected patients have a higher chance to develop pseudoexfoliation glaucoma (PEXG) (4). PEX is the most frequent reason for secondary open-angle glaucoma (5). Here, the high IOP is caused by an accumulation of pseudoexfoliation material in the anterior angle chamber, blocking the aqueous humor $(\mathrm{AH})$ outflow in the TM (6). Another form of OAG that develops independently from increased IOP is normal-tension glaucoma (NTG), as reviewed in (7). Here, glaucomatous damage can be observed despite what is generally considered as physiological IOP $(<21 \mathrm{mmHG})$. This implicates that disease mechanisms other than mere mechanical stress are likely to be involved in this subtype. One component adding to the possible disease mechanisms has been identified to be of an immunological kind. Signs of neuroinflammation and alterations in the innate immunity have been described in the context of glaucoma (8-12). One aspect here is also the diseasespecific alteration of the natural autoantibody repertoire, as has been shown in different studies over the past years (13-16). Several alterations in specific serological autoantibodies targeting ocular antigens have been recently identified. Thus, we wanted to further investigate whether the identified autoantibodies also show specifically altered levels in different subtypes of glaucoma, as already indicated in earlier studies of our research group (1719). To this end, we analyzed sera from POAG, NTG, and PEXG patients in comparison to a non-glaucomatous control group using protein microarray, also to identify potential markers that allow discrimination between OAG subtypes.

While the discovery of serological alterations of the autoantibody repertoire can be viable for diagnostic purposes when used as biomarkers, it tells us only little about their origin and function in the context of the disease. We wanted to look further into possible modes of action of autoantibodies that are altered in a disease specific manner. Thus, we also explored common characteristics of previously identified glaucomarelated antigens and investigate their relation to glaucoma pathogenesis. Autoantibodies to several ocular antigens have been frequently found by different groups using various methods, but they have not been regarded in a holistic approach. Most previous studies focus only on the identification of new markers but miss to put them in a context of disease mechanisms that allow new hypotheses to explain their role in OAG. To close this gap, we searched previously detected glaucoma-related antigens in the literature in addition to the one identified in this present study and analyzed their interactions and connections with the disease using bioinformatics tools.

\section{RESULTS}

\section{Analysis of Autoantibody Levels in Open-Angle Glaucoma Subtypes}

In this study, we used a set of 38 antigens associated with glaucoma and other neurodegenerative diseases for the preparation of antigen microarrays (Array design in Supplementary File 1). We analyzed the IgG autoantibody reactivity to these antigens in glaucoma patients, comprising NTG $(N=31)$, POAG $(N=43)$, and PEXG $(N=45)$, and a non-glaucomatous control group (CTRL; $N=46$ ). To identify alterations in antibody reactivity among these groups, we performed an ANOVA with consecutive post-hoc test (Tukey's HSD test for unequal $\mathrm{N}$ ). Figure 1A shows a heat map for the five significantly $(P<0.05)$ altered autoantibody levels in either group. Post-hoc testing revealed significant differences as follows: HTRA2 antibodies show significantly decreased levels in PEXG compared to CTRL $(P=0.022)$. Autoantibodies to HSP27 and CRYGS are significantly increased in PEXG compared to CTRL $(P<0.001)$ and POAG $(P=0.001)$. PEXG patients show decreased levels of CLTA/B/C autoantibodies compared to all other groups $(P<0.001)$. Group differences were not significant in the post-hoc test for MCM7. These results are also available in Supplementary File 2. Measurements of autoantibody reactivity to ACTA1, ACTN1, ADRB2, ATP5A1, DDX46, ENO1, FN1, MECP2, PKM2, PROK, SOD, TGFB1I1, TTR, and VIM were not possible because the protein spots did not pass quality requirements (maximum of $30 \%$ missing values) or signals were below the detection limit.

Using $k$ means clustering on all cases, three major clusters could be identified. Cluster 1 is mainly comprised $\left(X^{2} P<0.001\right)$ of POAG cases $(40.35 \%)$, Cluster 2 allows no classification of groups $\left(X^{2} P=0.958\right)$, whereas Cluster 3 mostly contains PEXG cases $\left(86.36 \%, X^{2} P<0.001\right.$ ) (See also Table 1 ). To investigate, which autoantibody levels have the biggest impact on the clustering, we used a feature selection algorithm (Statistical Feature Selection and Variable Screening) to find the best predictors. We identified the antibodies to Clathrin (CLTA/BC) as the most important predictor $\left(X^{2}=63.14 ; P<0.01\right)$ (Figure 1B). ANOVA and Tukey's HSD post-hoc test revealed that Clathrin autoantibody levels are significantly decreased in the serum of PEXG patients compared to the other groups (Figure 1C). An exemplary representation of the arrays of these autoantibodies is also depicted in Figure 1D.

\section{Correlation of Autoantibodies With Clinical Features}

Major hallmarks of glaucoma pathogenesis are IOP, visual field defects [measured as ("mean deviation" (MD)] and the cupping of the optic nerve that is assessed as the cup-disc ratio (CDR). We performed a Pearson correlation analysis to examine whether the serological antibody levels show an association with these clinical parameters (Table 2). The results reveal a weak negative correlation of CLTA/B/C autoantibodies with IOP $(r=-0.26)$ and a weak positive correlation with $\operatorname{CDR}(r=0.237)$. Further, a weak negative correlation of CRYGS autoantibodies $(r=-0.27)$ with CDR was revealed.

\section{Bioinformatics Analysis of Established Glaucoma-Related Autoantigens}

Starting with a literature search, we identified 28 antigens that have been identified as targets to glaucoma-related autoimmunity by different groups and methods (Table 3). To get an overall impression of the characteristics of theses previously identified glaucoma-related antigens, we conducted a protein-protein interaction analysis, as well as a GO term 

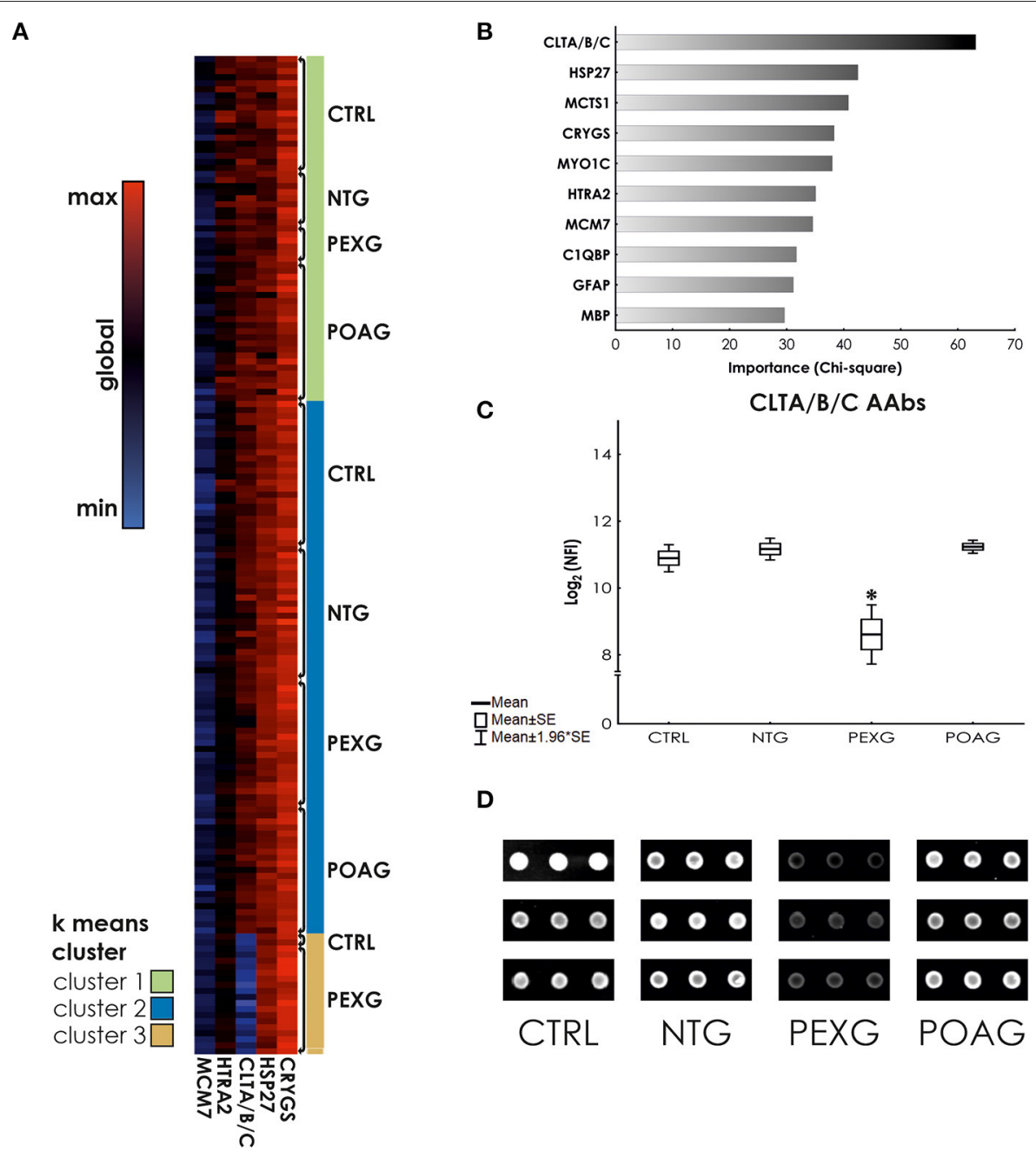

FIGURE 1 | K means clustering and predictor screening. (A) Heat map showing autoantibody reactivities in glaucoma subtypes and control samples. (B) Predictor screening of autoantibody levels. The most important predictor for group affiliation are CLTA/B/C antibody levels. (C) ANOVA with HSD unequal N post-hoc test. CLTA/B/C autoantibody levels are significantly decreased in serum of PEXG patients compared to the other groups. (D) Representative examples of CLTA/B/C antibody reactivity on processed microarrays of individual samples. ${ }^{*}$ Indicates statistical significance at $P<0.05$.

TABLE 1 | Distribution of glaucoma cases and controls in the three $k$ means clusters.

\begin{tabular}{|c|c|c|c|c|c|}
\hline$\%$ & CTRL & NTG & PEXG & POAG & Sum \\
\hline Cluster 1 & 33.33 & 15.79 & 10.53 & 40.35 & 100 \\
\hline Cluster 2 & 27.27 & 25 & 23.86 & 23.86 & 100 \\
\hline Cluster 3 & 10 & 0 & 90 & 0 & 100 \\
\hline Cases & 27.27 & 18.79 & 27.27 & 26.67 & 100 \\
\hline
\end{tabular}

Cluster 1 mainly contains cases of the POAG group (40.35\%). Cluster 2 is equally separated among the four groups. Cluster 3 predominantly contains PEXG cases (90\%). $X^{2}$-test is

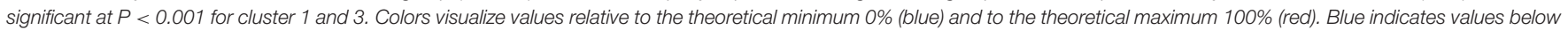
the expected value, red indicates values above the expected value. Light colors represent values close to the expected value.

enrichment analysis using Metascape. We found that 22 of the 28 antigens had at least one interaction partner among the tested proteins (Figure 2A). A strong network was identified by Metascapes' Molecular Complex Detection algorithm (MCODE) with exceptionally high number of interactions for six of the antigens. These antigens are HSPA1A, HSPD1, YWHAZ, ENO2, PGAM1, and VDAC2. The GO enrichment analysis for all 28 antigens revealed 13 terms describing biological processes as significantly enriched. The most significantly enriched processes were regulation of mRNA process, protein 
TABLE 2 | Pearson correlation analysis.

\begin{tabular}{lccc}
\hline & IOP $(\boldsymbol{N}=\mathbf{1 6 0})$ & MD $(\boldsymbol{N}=\mathbf{7 7})$ & CDR $(\boldsymbol{N}=\mathbf{1 1 3})$ \\
\hline HTRA2 & -0.088 & -0.073 & 0.042 \\
MCM7 & -0.082 & 0.112 & 0.170 \\
HSP27 & 0.077 & -0.111 & -0.175 \\
CRYGS & 0.024 & -0.195 & -0.270 \\
CLTA/B/C & -0.255 & 0.037 & 0.237 \\
\hline
\end{tabular}

Table shows correlation coefficients ( $r$ ). Marked entries (red) are significant at $P<0.05$. Analysis was carried out with all available cases.

folding, blood coagulation and apoptosis (Figure 2B). The GO enrichment analysis for the MCODE cluster of antigens only, revealed a significant enrichment of three biological processes. These comprise apoptotic mitochondrial changes, nucleobasecontaining catabolic processes and heterocycle catabolic processes (Figure 2C). Regarding the cellular components of the MCODE cluster of antigens, three compartments were enriched (Table 4). Four of the six antigens are located in the myelin sheath and/or in mitochondria. Also, 50\% of these antigens are to be found in the extracellular space.

\section{DISCUSSION}

In this study, we analyzed serological autoantibodies in sera of OAG patients and non-glaucomatous controls by means of an antigen microarray approach. We found altered levels of antibodies to CLTA/B/C, CRYGS, HSP27, and HTRA2 using ANOVA. Although the Pearson correlation analysis revealed few significant correlations between some autoantibody levels and glaucoma hallmarks, the relationships are very weak. With a correlation coefficient of $|r|<0.3$ the correlations can be considered negligible. Thus, it can be concluded that the abundance of the analyzed antibodies is not essentially dependent on alterations of IOP, MD or CDR and vice versa. Although, some biological processes involved in pathological changes influencing IOP, and advancing neurodegeneration leading to increasing CDRs could have stronger dependencies leading to the observed statistical significance, but this cannot be deduced from the data at hand.

We analyzed, whether these autoantibodies might serve as disease markers to classify non-glaucomatous subjects and OAG subtypes. An earlier study of our research group already found significant differences between the antibody profiles of POAG and NTG, also in comparison to a control group, indicating their diagnostic value for OAG subtype discrimination (19). Another previous study showed significant differences between the antibody profiles to retinal antigens in aqueous humor of patients with PEXG and a control group (17). This study also compared the antibody profiles of POAG and PEXG patients. Although these two forms of OAG have vast differences in their pathogenesis, they showed no significant differences in their antibody profiles between each other. For a potential diagnostic discrimination of various glaucoma subtypes, suitable biomarker candidates need to be identified. Here, we achieved to find a
TABLE 3 | Glaucoma-related antigens identified in different previous studies.

\begin{tabular}{|c|c|c|}
\hline Protein & Gene ID & References \\
\hline HSP60 & HSPD1 & (20) \\
\hline HSP27 & HSPB1 & $(13,21)$ \\
\hline HSP70 & HSPA1 & (22) \\
\hline Alpha A-crystallin & CRYAA & (21) \\
\hline Alpha B-crystallin & CRYAB & (22) \\
\hline$\beta$-L-crystallin & CRYBA1 & (13) \\
\hline Annexin 5 & ANXA5 & (13) \\
\hline Ubiquitin & $\begin{array}{l}\text { Isoform not specified; UBB used } \\
\text { for analysis }\end{array}$ & (13) \\
\hline Glial fibrillary acidic protein & GFAP & $(13,18)$ \\
\hline $14-3-3$ & $\begin{array}{l}\text { Isoform not specified; YWHAZ } \\
\text { used for analysis }\end{array}$ & (23) \\
\hline Alpha fodrin & SPTAN1 & (24) \\
\hline Gamma enolase & ENO2 & $(25,26)$ \\
\hline Vimentin & VIM & (22) \\
\hline Myelin basic protein & MBP & $(13,19)$ \\
\hline Retinaldehyde-binding protein & RLBP1 & (18) \\
\hline Glutathion-S-transferase & GST & (27) \\
\hline Retinal S-antigen & SAG & (18) \\
\hline Histone $\mathrm{H} 4$ & $\mathrm{H} 4$ & (18) \\
\hline Alpha 1 antitrypsin & SERPINA1 & (13) \\
\hline Gamma synuclein & SNCG & $\begin{array}{l}\text { Claimed in } \\
\text { (28) }\end{array}$ \\
\hline $\begin{array}{l}\text { Voltage-dependent } \\
\text { anion-selective channel protein } 2\end{array}$ & VDAC2 & (14) \\
\hline Caldesmon & CALD1 & (14) \\
\hline Phosphoglycerate mutase 1 & PGAM1 & (14) \\
\hline $\begin{array}{l}\text { Threonine-tRNA ligase 1, } \\
\text { cytoplasmic }\end{array}$ & TARS1 & (16) \\
\hline $\begin{array}{l}\text { Complement component } 1 \mathrm{Q} \\
\text { subcomponent-binding protein, } \\
\text { mitochondrial }\end{array}$ & C1QBP & (16) \\
\hline Paraneoplastic antigen Ma2 & PNMA2 & (16) \\
\hline Beta-2 adrenergic receptor & ADRB2 & (29) \\
\hline Clathrin & CLTA/B/C & This study \\
\hline
\end{tabular}

putative autoantibody biomarker that shows significantly altered serological levels in PEXG compared to the other most common OAG types, POAG and NTG. Using a predictor screening algorithm, we identified antibodies to CLTA/B/C (Clathrin) as the most important predictor.

Clathrin is a protein majorly involved in the formation of vesicles associated with clathrin-mediated endocytosis [reviewed in (30)]. Not much is known about the association of clathrin with glaucoma. However, northern blot analysis of gene expression patterns in PEX patients has shown an increase in clathrin expression in the lens epithelium (31). Generally, it has been discussed that uptake of circulating antibodies is conveyed by clathrin-mediated endocytosis that opens the opportunity of autoantibodies to cause various effects on an intracellular level $(32,33)$. Antibodies to clathrin, however, have not been reported in relation to PEXG. Circulating antibodies are hypothetically 


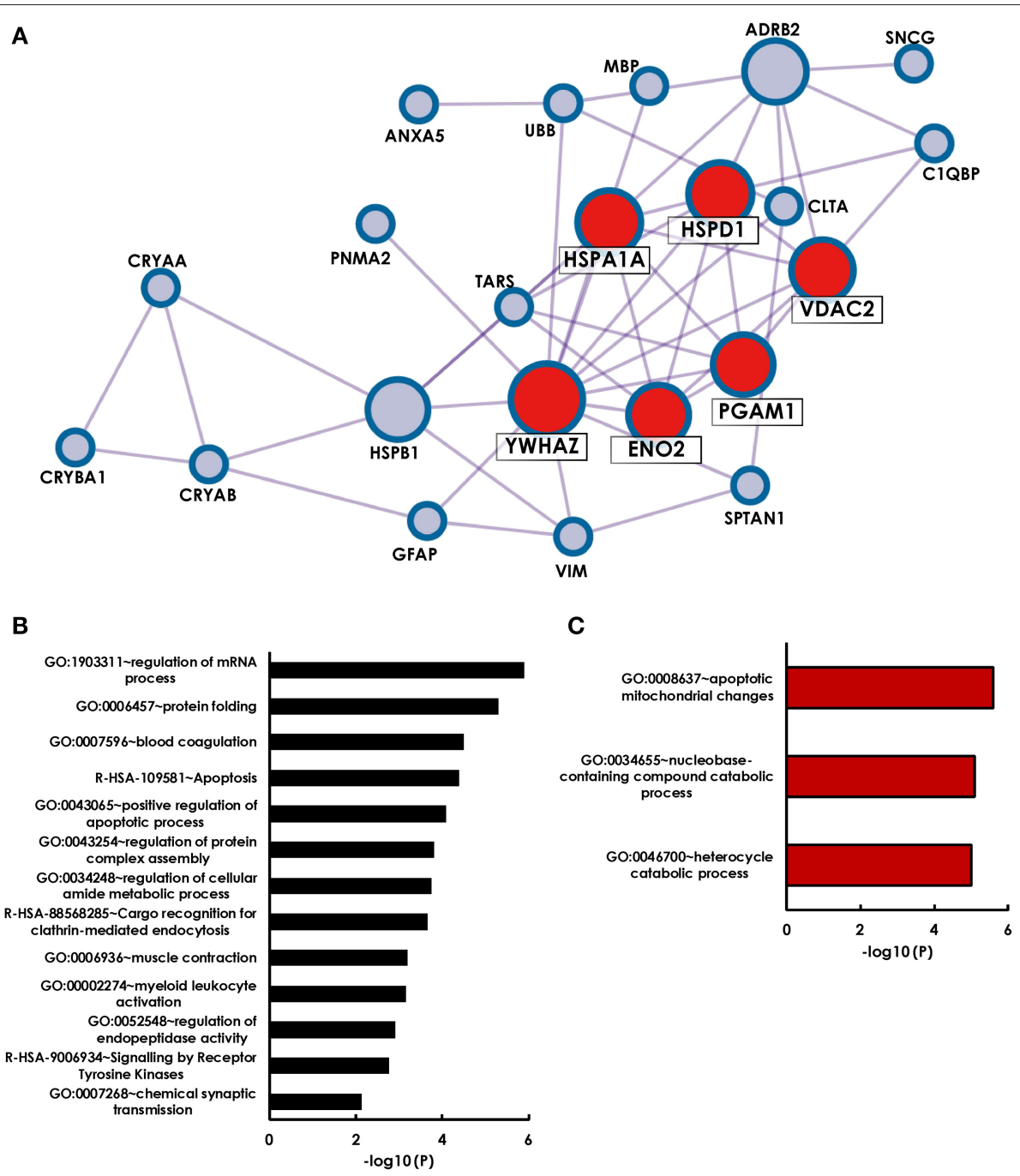

FIGURE 2 | Holistic bioinformatics analysis of previously established glaucoma-related autoantigens. (A) Protein-protein interaction network. Twenty-two of the twenty-eight glaucoma-related antigens have at least one interaction partner among each other. Especially strong interactions were observed for six antigens (HSPA1A, HSPD1, YWHAZ, VDAC2, PGAM1, ENO2), that were identified by the Metaspcape algorithm MCODE (marked with red spots). (B) GO analysis of 28 glaucoma-related antigens. Shown are significantly enriched GO terms of biological processes and reactome gene sets. (C) GO analysis of MCODE cluster antigens only.

TABLE 4 | Cellular components of MCODE clustered antigens.

\begin{tabular}{llccclcccc}
\hline GO term & Cellular component & Count & $\%$ & P-value & Antigens & List total & Pop hits & Pop total & Fold enrichment \\
\hline GO:0043209 & Myelin sheath & 4 & 66.67 & $<0.001$ & ENO2, PGAM1, HSPD1, VDAC2 & 6 & 152 & 18,224 \\
GO:0005739 & Mitochondrion & 4 & 66.67 & 0.003 & YWHAZ, HSPA1A, HSPD1, VDAC2 & 6 & 1,331 & 18,224 & 9.128 \\
GO:0005615 & Extracellular space & 3 & 50 & 0.047 & YWHAZ, ENO2, HSPD1 & 6 & 1,347 & 18,224 & 6.765 \\
\hline
\end{tabular}

DAVID GO enrichment analysis.

attributed to many different functions, including agonistic and antagonistic receptor effects or activation of the complement cascade (34). Stimulating as well as inhibitory effects are possible. The aggregation of exfoliation material in the anterior chamber has vision-threatening effects in PEX patients. Polymorphisms in the LOXL1 gene contribute to the accumulation of elastic myofibrils and extracellular matrix that impair the physiological function of the TM, leading to increased IOP and severe damage to the optic nerve as a consequence (35). Possibly, an altered clathrin-mediated extracellular matrix turnover in PEXG patients plays a role in the pathogenesis. Thus, the decreased autoantibody levels might be a consequence or precursor of this condition. Further studies are necessary to investigate the causalities of clathrin and anti-clathrin antibodies in PEXG. 
Although perturbations in levels of serological antibodies might hint toward disease-related alterations in quality or quantity of the corresponding autoantigen, the conclusions one can draw of this remain vague. It is also not possible to deduce possible disease-related effects merely of the levels of single specific antibodies. Therefore, it is important to evaluate changes in the serological antibody repertoire not isolated, but in a holistic approach, using all available information. Thus, we took one step further and analyzed the entirety of known OAG-related autoantibodies to identify commonalities and biological processes that are the main crossroads for the immunological contribution in OAG. Autoantibodies to ocular antigens that show altered levels in glaucoma patients have already been described by numerous different studies conducted by various research groups. To learn more about the nature of the autoantigens identified in the past and in this present study, we implemented a holistic bioinformatics analysis to investigate the relations between these glaucoma antigens. We assumed that the examination of how they connect to each other in the context of the disease could help for a better understanding of synergies between autoimmune effects and other disease mechanisms. We found 13 biological processes enriched among the glaucoma-related antigens. Proteins were most highly enriched in regulation of mRNA processes, followed by protein folding, blood coagulation and apoptosis. Pathological changes in these processes were found in OAG patients and we assume a glaucoma-related association between alterations of these processes and corresponding autoantibody levels. Deregulations of RNA metabolism associated processes are a common feature of neurodegenerative diseases (36). Alterations in the regulation of some glaucoma-related mRNAs were also reported in TM cells of glaucoma patients (37), showing a possible relation to altered autoantibody abundance. The enrichment of antigens that are part of the biological process of protein folding arises mainly from overrepresentation of molecular chaperons. Disturbed protein folding can cause formation of protein aggregates that, when accumulating, can induce the unfolded protein response (UPR), which protects the cell from endoplasmic reticulum (ER) stress (38). ER stress in turn can lead to apoptosis if the capacities of the UPR are exceeded. ER stress-induced apoptosis is common in neurodegenerative diseases and also occurs in glaucoma pathogenesis (39). Abnormal blood coagulation was described in POAG patients in form of age-dependent spontaneous platelet aggregation (40) and platelet activation was also assumed to be involved in glaucoma pathomechanisms (41). Glaucoma-related autoantibodies were shown to especially reflect the platelet-derived growth factor receptor pathway (16). Whether the detected autoantibodies partake in the deregulation of these processes or act as possible countermeasures cannot be answered here. We can however assume a strong influence of these alterations on the serological autoantibody repertoire, presumably reflecting the antigenic status.

Findings from the PPI network suggest a special role in glaucoma associated immunity for the six antigens clustered by the MCODE algorithm. These antigens are mainly associated with mitochondria and apoptotic mitochondrial changes. In general, apoptosis is considered the main mechanism of
RGC demise in glaucoma (42) and previous studies already emphasized the importance of mitochondrial dysfunction as an aspect in glaucoma pathogenesis, which is also considered a major cause of cell death of RGCs $(43,44)$. Pro-apoptotic alterations in the mitochondrial proteome have already been observed in retinal cells after incubation with serum of POAG patients (45). This indicates that serological autoantibodies are capable to promote disease progression and persistent neuronal damage. Future work should further investigate the connection and molecular mechanisms of a possible antibody-mediated impairment of mitochondria in RGCs.

Additionally, four of the six MCODE clustered antigens are also located in myelin sheaths. Conventionally, glaucoma is not considered as a demyelinating disorder. Recent investigations however found evidence that demyelination could play a role in the mechanisms causing neuronal damage (46). Although there is some inconsistency in data, suggesting that this could not be true for all cases (47). Additionally, a study in DBA/2J mice has shown that early insults of the optic nerve occur in proximity to the lamina cribrosa and that axon segments in the lamina and more distal undergo degeneration (48). Retinal ganglion cells are unmyelinated until they pass through the lamina cribrosa, but the myelinated axons beyond might be targeted by glaucoma-related antibodies. Autoantibodies to proteins of the myelin sheath are also known form other diseases such as multiple sclerosis and neuromyelitis optica. Here, antibodies against myelin-associated proteins [e.g., antimyelin oligodendrocyte glycoprotein, anti-myelin basic protein (49) and anti-aquaporin 4] are frequently found in patients. Their role in the pathogenesis is not finally clarified yet. Though it is assumed that they have the potential to inflict axonal damage and inflammatory demyelination, which would also apply to the optic nerve cells distal to the lamina cribrosa $(49,50)$. This gives rise to the hypothesis that damages of the optic nerve sheath mediated by antibodies could also be capable to cause neuronal damage in glaucoma. On the other hand, IgG autoantibodies have been described to be involved in the debris clearance after injury of neuronal cells of the peripheral nerve system, thereby supporting axon regeneration (51). However, regardless of the effect of the IgG class autoantibodies, their occurrence is strongly linked to injuries of nerve cells and their axons. The antibody-mediated effects affecting glaucoma pathogenesis have not yet been elucidated and will require further investigation in future studies.

While our study gives new insights into autoantibodies in glaucoma, it also has some limitations that should be acknowledged. The detection of decreased levels of CLTA/B/C antibodies in serum of PEXG patients was conducted by protein microarrays as sole method. A validation with additional methods would increase the significance of this discovery. Also, more experiments are needed to elucidate the biological mechanisms behind this finding. This monocentric study is further limited by its relatively small sample size and unequal group size, which we attempted to deal with by using significance tests for unequal $N$. Furthermore, it will be interesting to see, whether patients suffering from pseudoexfoliation syndrome without glaucoma also exhibit lower levels of clathrin antibodies. 
TABLE 5 | Demographics of study population.

\begin{tabular}{llll}
\hline & $\boldsymbol{N}$ & $\mathbf{m} / \mathbf{f}$ & Age (min-max) \\
\hline CTRL & 46 & $27 / 19$ & $66.96(34-83)$ \\
NTG & 31 & $15 / 16$ & $68.29(49-80)$ \\
PEXG & 45 & $21 / 24$ & $70.47(52-86)$ \\
POAG & 43 & $25 / 18$ & $66.37(24-81)$ \\
\hline
\end{tabular}

Nevertheless, this marker can be useful to distinguish PEXG from other forms of glaucoma.

Overall, these results contribute to the assumption that serological autoantibodies can reflect pathological changes in the affected sites of the eye. Glaucoma-related autoantigens are enriched in biological processes with a strong link to previously described pathomechanisms. The causality of autoantibody genesis and corresponding pathological events, however, still remains a conundrum. Even though the molecular mechanisms of autoantibodies in glaucoma cannot be revealed in our study, it delivers enough evidence to justify future studies investigating the interplay of the immune system and glaucoma in the here identified biological processes in more detail. Apart from that, the observation of IgG level alterations in serum can be exploited for the monitoring of the disease and usage for diagnostic purposes, nonetheless. The detection of significantly altered autoantibody levels to clathrin promotes prospective diagnostic glaucoma-subtyping, especially for the discrimination of PEXG from other forms of OAG.

\section{MATERIALS AND METHODS}

\section{Sera}

Sample collection was carried out in accordance with the Declaration of Helsinki on biomedical research involving human subjects. Written informed consent was obtained from each subject. The study was approved by the ethics committee of the Landesärztekammer Rheinland-Pfalz. All subjects included in this study received an ophthalmic examination at the department of ophthalmology of the university medical center in Mainz, Germany. Glaucoma patients were diagnosed according to the guidelines of the European Glaucoma Society (52). Visual field defects were determined using OCTOPUS 101 Perimeter (HaagStreit, Wedel, Germany) or Humphrey Visual Field analyzer (Carl Zeiss Meditec, Dublin, CA). Correlation analysis was only run with OCTOPUS data sets, to avoid systematic bias. The control group consists of non-glaucomatous subjects. According to the anamnestic query, none of the included subjects suffered from Alzheimer's or Parkinson's disease. One subject of the non-glaucomatous control group reported suffering from multiple sclerosis. Glaucoma patients were diagnosed based on glaucomatous optic disc damage and typical visual field defects. All patients had open iridiocorneal angles. PEXG was diagnosed when patients showed PEX material in at least on eye. PEXG and POAG patients showed an IOP $>21 \mathrm{mmHg}$, while IOP in NTG patients was $<21 \mathrm{mmHg}$. The demographics of the study population can be found in Table 5 .

\section{Antigen Microarray Analysis}

Recombinant or purified proteins selected for the antigen microarray are listed in Supplementary File 1. The arrays were produced in our lab, as described elsewhere (14). Array hybridization was performed using 16-well incubation chambers (ProPlate Multiwell chambers, Grace Biolabs, Bend, USA). All incubation steps were carried out on an orbital shaker at $4^{\circ} \mathrm{C}$. Arrays were incubated for $1 \mathrm{~h}$ with a blocking buffer (Super G, Grace Biolabs, Bend, Oregon, USA). Then, the blocking buffer was removed, and the slides were washed three times with phosphate-buffered saline containing 0.5\% Tween-20 (PBST). Subsequently, the arrays were incubated with $100 \mu \mathrm{L}$ serum in a 1:250 in PBS overnight. As negative control PBS only arrays were included on each slide. Next, slides were washed three times again with PBST followed by incubation with an antihuman antibody conjugated with a fluorophore (Alexa Fluor ${ }^{\circledR}$ 647 AffiniPure Goat Anti-Human IgG, Fc $\gamma$ fragment specific, 109-605-008, Jackson Immunoresearch) as secondary antibody diluted 1:500 in PBS for $1 \mathrm{~h}$. Next, the arrays were washed twice with PBST and twice with ultrapure water. Lastly, the slides were dried for $2 \mathrm{~min}$ in a vacuum centrifuge concentrator (SpeedVac, Thermo Scientific, Waltham, Massachusetts, USA).

Array images were acquired as 16-bit TIF file using a high-resolution confocal laser scanner (428 Array Scanner, Affymetrix, Santa Clara, California, USA). The image analysis software Imagene (Imagene 5.5, BioDiscovery Inc., Los Angeles, California, USA) has been used to quantify spot intensities. Poor quality spots have been manually flagged and removed from the analysis.

\section{Microarray Data Pre-procession}

Net signal intensities were calculated by subtraction of local background intensity. Signals reaching negative values after background subtraction were treated as missing data. Negative control signal intensities were subtracted from each spot. Intensities from the triplicate spots were averaged, yielding one mean fluorescence intensity. All signals were then normalized to the IgG control spots included on each subarray by median centring to reduce intra-slide variability and batch effects. Therefore, IgG median signal intensities were divided by the overall IgG signal median to obtain a factor for each subarray. All further analyses are based on these normalized fluorescence intensities (NFI). To ensure the robustness of the dataset and reduce the influence of outliers, values below the 5 th and above the 95th percentile in each group were set as missing data. Only data above the defined limit of detection (mean fluorescence intensity from the negative control of each antigen + standard deviation) were eligible. Targets with overall more than $30 \%$ missing data were not eligible for statistical analyses. Missing data of targets with $<30 \%$ missing values was imputed using the $k$ nearest-neighbor (KNN) algorithm. Grand mean normalization was applied for all arrays.

\section{Statistical Analysis}

Statistical analyses were performed using Statistica (Statistica 13, Statsoft, Tulsa, Oklahoma, USA). One-way ANOVA with consecutive Tukey's HSD post-hoc test for unequal $\mathrm{n}$ was 
performed to determine significant alterations in antibody levels among the study groups. Pearson's correlation coefficients were used to evaluate correlations of autoantibody levels and clinical parameters. A $P$-value of $<0.05$ was considered statistically significant. Heat mapping and $\mathrm{k}$ means clustering was implemented using Morpheus (https://software.broadinstitute. org/morpheus/).

\section{Protein-Protein Interaction Networks and Gene Ontology Enrichment Analysis}

Gene Ontology enrichment analysis and the protein-protein interaction network was conducted in Metascape (http:// metascape.org) (53). Also, Metascape's molecular complex detection (MCODE) algorithm was used to detect densely connected network components.

\section{DATA AVAILABILITY STATEMENT}

The raw data supporting the conclusions of this article will be made available by the authors, without undue reservation.

\section{ETHICS STATEMENT}

The studies involving human participants were reviewed and approved by Ethics Committee of the Landesärztekammer

\section{REFERENCES}

1. Montecchi-Palmer M, Bermudez JY, Webber HC, Patel GC, Clark AF, Mao W. TGFbeta 2 induces the formation of cross-linked actin networks (CLANs) in human trabecular meshwork cells through the smad and nonsmad dependent pathways. Invest Ophthalmol Vis Sci. (2017) 58:1288-95. doi: 10.1167/iovs.16-19672

2. Zhavoronkov A, Izumchenko E, Kanherkar RR, Teka M, Cantor C, Manaye $\mathrm{K}$, et al. Pro-fibrotic pathway activation in trabecular meshwork and lamina cribrosa is the main driving force of glaucoma. Cell Cycle. (2016) 15:1643-52. doi: 10.1080/15384101.2016.1170261

3. Sacca SC, Pulliero A, Izzotti, A. The dysfunction of the trabecular meshwork during glaucoma course. J Cell Physiol. (2015) 230:510-25. doi: $10.1002 /$ jcp. 24826

4. Mitchell P, Wang JJ, Hourihan F. The relationship between glaucoma and pseudoexfoliation: the Blue Mountains Eye Study. Arch Ophthalmol. (1999) 117:1319-24. doi: 10.1001/archopht.117.10.1319

5. Naumann GO, Schlotzer-Schrehardt U, Kuchle M. Pseudoexfoliation syndrome for the comprehensive ophthalmologist. Intraocular and systemic manifestations. Ophthalmology. (1998) 105:951-68. doi: 10.1016/S0161-6420(98)96020-1

6. Ritch R. Ocular findings in exfoliation syndrome. J Glaucoma. (2018) 27(Suppl. 1):S67-71. doi: 10.1097/IJG.0000000000000986

7. Esporcatte BL, Tavares IM. Normal-tension glaucoma: an update. Arq Bras Oftalmol. (2016) 79:270-6. doi: 10.5935/0004-2749.20160077

8. Ten Berge JC, Fazil Z, van den Born I, Wolfs RCW, Schreurs MWJ, Dik WA, et al. Intraocular cytokine profile and autoimmune reactions in retinitis pigmentosa, age-related macular degeneration, glaucoma and cataract. Acta Ophthalmol. (2018) 97:185-92 doi: 10.1111/aos.13899

9. Williams PA, Marsh-Armstrong N, Howell GR, Lasker/IRRF Initiative on Astrocytes and Glaucomatous Neurodegeneration Participants. Neuroinflammation in glaucoma: a new opportunity. Exp Eye Res. (2017) 157:20-7. doi: 10.1016/j.exer.2017.02.014

10. Bell K, Holz A, Ludwig K, Pfeiffer N, Grus FH. Elevated regulatory T Cell levels in glaucoma patients in comparison to healthy controls. Curr Eye Res. (2017) 42:562-7. doi: 10.1080/02713683.2016.1205629
Rheinland-Pfalz. The patients/participants provided their written informed consent to participate in this study.

\section{AUTHOR CONTRIBUTIONS}

VB planned the experimental design of this study, performed bioinformatics and microarray analysis, interpreted the data, and wrote the manuscript. FG critically revised the manuscript. FG and NP contributed to conception of the study and provided resources. All authors contributed to the article and approved the submitted version.

\section{FUNDING}

This work was supported by the German Federal Ministry of Education and Research (Bundesministerium für Bildung und Forschung); KMU-innovativ: Medizintechnik (FKZ: 13GW0193B).

\section{SUPPLEMENTARY MATERIAL}

The Supplementary Material for this article can be found online at: https://www.frontiersin.org/articles/10.3389/fimmu. 2021.616421/full\#supplementary-material

11. Tezel G, Yang X, Luo C, Kain AD, Powell DW, Kuehn MH, et al. Oxidative stress and the regulation of complement activation in human glaucoma. Invest Ophthalmol Vis Sci. (2010) 51:5071-82. doi: 10.1167/iovs.10-5289

12. Reinehr S, Reinhard J, Gandej M, Kuehn S, Noristani R, Faissner A, et al. Simultaneous complement response via lectin pathway in retina and optic nerve in an experimental autoimmune glaucoma model. Front Cell Neurosci. (2016) 10:140. doi: 10.3389/fncel.2016.00140

13. Boehm N, Wolters D, Thiel U, Lossbrand U, Wiegel N, Pfeiffer N, et al. New insights into autoantibody profiles from immune privileged sites in the eye: a glaucoma study. Brain Behav Immun. (2012) 26:96-102. doi: 10.1016/j.bbi.2011.07.241

14. Beutgen VM, Perumal N, Pfeiffer N, Grus FH. Autoantibody biomarker discovery in primary open angle glaucoma using serological proteome analysis (SERPA). Front Immunol. (2019) 10:381. doi: 10.3389/Fimmu.2019.00381

15. Tezel G, Thornton IL, Tong MG, Luo C, Yang X, Cai J, et al. Immunoproteomic analysis of potential serum biomarker candidates in human glaucoma. Invest Ophthalmol Vis Sci. (2012) 53:8222-31. doi: 10.1167/iovs.12-10076

16. Beutgen VM, Schmelter C, Pfeiffer N, Grus FH. Autoantigens in the trabecular meshwork and glaucoma-specific alterations in the natural autoantibody repertoire. Clin Transl Immunology. (2020) 9:e01101. doi: 10.1002/cti2.1101

17. Joachim SC, Wuenschig D, Pfeiffer N, Grus FH. IgG antibody patterns in aqueous humor of patients with primary open angle glaucoma and pseudoexfoliation glaucoma. Mol Vis. (2007) 13:1573-9.

18. Reichelt J, Joachim SC, Pfeiffer N, Grus FH. Analysis of autoantibodies against human retinal antigens in sera of patients with glaucoma and ocular hypertension. Curr Eye Res. (2008) 33:253-61. doi: 10.1080/02713680701871157

19. Joachim SC, Reichelt J, Berneiser S, Pfeiffer N, Grus FH. Sera of glaucoma patients show autoantibodies against myelin basic protein and complex autoantibody profiles against human optic nerve antigens. Graefes Arch Clin Exp Ophthalmol. (2008) 246:573-80. doi: 10.1007/s00417-0070737-8

20. Wax MB, Tezel G, Saito I, Gupta RS, Harley JB, Li Z, et al. Anti-Ro/SS-A positivity and heat shock protein antibodies in patients with normal-pressure glaucoma. Am J Ophthalmol. (1998) 125:145-57. 
21. Tezel G, Seigel GM, Wax MB. Autoantibodies to small heat shock proteins in glaucoma. Invest Ophthalmol Vis Sci. (1998) 39:2277-87.

22. Joachim SC, Bruns K, Lackner KJ, Pfeiffer N, Grus FH. Antibodies to alpha B-crystallin, vimentin, and heat shock protein 70 in aqueous humor of patients with normal tension glaucoma and IgG antibody patterns against retinal antigen in aqueous humor. Curr Eye Res. (2007) 32:501-9. doi: 10.1080/02713680701375183

23. Bell K, Wilding C, Funke S, Pfeiffer N, Grus FH. Protective effect of 14-33 antibodies on stressed neuroretinal cells via the mitochondrial apoptosis pathway. BMC Ophthalmol. (2015) 15:64. doi: 10.1186/s12886-015-0044-9

24. Grus FH, Joachim SC, Bruns K, Lackner KJ, Pfeiffer N, Wax MB. Serum autoantibodies to alpha-fodrin are present in glaucoma patients from Germany and the United States. Invest Ophthalmol Vis Sci. (2006) 47:968-76. doi: 10.1167/iovs.05-0685

25. Maruyama I, Ohguro H, Ikeda Y. Retinal ganglion cells recognized by serum autoantibody against gamma-enolase found in glaucoma patients. Invest Ophthalmol Vis Sci. (2000) 41:1657-65.

26. Ikeda Y, Maruyama I, Nakazawa M, Ohguro H. Clinical significance of serum antibody against neuron-specific enolase in glaucoma patients. Jpn J Ophthalmol. (2002) 46:13-7. doi: 10.1016/s0021-5155(01)00455-5

27. Yang J, Tezel G, Patil RV, Romano C, Wax MB. Serum autoantibody against glutathione S-transferase in patients with glaucoma. Invest Ophthalmol Vis Sci. (2001) 42:1273-6.

28. Wilding C, Bell K, Beck S, Funke S, Pfeiffer N, Grus FH. GammaSynuclein antibodies have neuroprotective potential on neuroretinal cells via proteins of the mitochondrial apoptosis pathway. PLoS ONE. (2014) 9:e90737. doi: 10.1371/journal.pone.0090737

29. Junemann A, Hohberger B, Rech J, Sheriff A, Fu Q, Schlotzer-Schrehardt $\mathrm{U}$, et al. Agonistic Autoantibodies to the beta2-adrenergic receptor involved in the pathogenesis of open-angle glaucoma. Front Immunol. (2018) 9:145. doi: 10.3389/fimmu.2018.00145

30. Mettlen M, Chen PH, Srinivasan S, Danuser G, Schmid SL. Regulation of clathrin-mediated endocytosis. Annu Rev Biochem. (2018) 87:871-96. doi: 10.1146/annurev-biochem-062917-012644

31. Zenkel M, Poschl E, von der Mark K, Hofmann-Rummelt C, Naumann GO, Kruse FE, et al. Differential gene expression in pseudoexfoliation syndrome. Invest Ophthalmol Vis Sci. (2005) 46:3742-52. doi: 10.1167/iovs.05-0249

32. Goldstein JL, Anderson RG, Brown MS. Coated pits, coated vesicles, and receptor-mediated endocytosis. Nature. (1979) 279:679-85. doi: $10.1038 / 279679 \mathrm{a} 0$

33. Congdon EE, Gu J, Sait HB, Sigurdsson EM. Antibody uptake into neurons occurs primarily via clathrin-dependent Fcgamma receptor endocytosis and is a prerequisite for acute tau protein clearance. J Biol Chem. (2013) 288:3545265. doi: 10.1074/jbc.M113.491001

34. Diamond B, Huerta PT, Mina-Osorio P, Kowal C, Volpe BT. Losing your nerves? Maybe it's the antibodies. Nat Rev Immunol. (2009) 9:449-56. doi: $10.1038 /$ nri2529

35. Schlotzer-Schrehardt U. Molecular pathology of pseudoexfoliation syndrome/glaucoma-new insights from LOXL1 gene associations. Exp Eye Res. (2009) 88:776-85. doi: 10.1016/j.exer.2008.08.012

36. Liu EY, Cali CP, Lee EB. RNA metabolism in neurodegenerative disease. Dis Model Mech. (2017) 10:509-18. doi: 10.1242/dmm.028613

37. Mochizuki H, Murphy CJ, Brandt JD, Kiuchi Y, Russell P. Altered stability of mRNAs associated with glaucoma progression in human trabecular meshwork cells following oxidative stress. Invest Ophthalmol Vis Sci. (2012) 53:1734-41. doi: 10.1167/iovs.12-7938

38. Anholt RR, Carbone MA. A molecular mechanism for glaucoma: endoplasmic reticulum stress and the unfolded protein response. Trends Mol Med. (2013) 19:586-93. doi: 10.1016/j.molmed.2013.06.005

39. Peters JC, Bhattacharya S, Clark AF, Zode GS. Increased endoplasmic reticulum stress in human glaucomatous trabecular meshwork cells and tissues. Invest Ophthalmol Vis Sci. (2015) 56:3860-8. doi: $10.1167 /$ iovs.14-16220

40. Hoyng PF, de Jong N, Oosting H, Stilma J. Platelet aggregation, disc haemorrhage and progressive loss of visual fields in glaucoma. A seven year follow-up study on glaucoma. Int Ophthalmol. (1992) 16:65-73. doi: 10.1007/BF00918934

41. Ma Y, Han J, Li S, Zhang A, Cao W, Sun X. Association between platelet parameters and glaucoma severity in primary open-angle glaucoma. $J$ Ophthalmol. (2019) 2019:3425023. doi: 10.1155/2019/3425023

42. Guo L, Moss SE, Alexander RA, Ali RR, Fitzke FW, Cordeiro MF. Retinal ganglion cell apoptosis in glaucoma is related to intraocular pressure and IOP-induced effects on extracellular matrix. Invest Ophthalmol Vis Sci. (2005) 46:175-82. doi: 10.1167/iovs.04-0832

43. Yang XJ, Ge J, Zhuo YH. Role of mitochondria in the pathogenesis and treatment of glaucoma. Chin Med J (Engl). (2013) 126:4358-65. doi: 10.3760/cma.j.issn.0366-6999.20131956

44. Osborne NN, Nunez-Alvarez C, Joglar B, Del Olmo-Aguado S. Glaucoma: focus on mitochondria in relation to pathogenesis and neuroprotection. Eur J Pharmacol. (2016) 787:127-33. doi: 10.1016/j.ejphar.2016.04.032

45. Bell K, Funke S, Pfeiffer N, Grus FH. Serum and antibodies of glaucoma patients lead to changes in the proteome, especially cell regulatory proteins, in retinal cells. PLoS ONE. (2012) 7:e46910. doi: 10.1371/journal.pone.0046910

46. You Y, Joseph C, Wang C, Gupta V, Liu S, Yiannikas C, et al. Demyelination precedes axonal loss in the transneuronal spread of human neurodegenerative disease. Brain. (2019) 142:426-42. doi: 10.1093/brain/awy338

47. Graham SL. The effects of glaucoma on the latency of the multifocal visual evoked potential. Br J Ophthalmol. (2006) 90:1077-8. doi: 10.1136/bjo.2006.097592

48. Howell GR, Libby RT, Jakobs TC, Smith RS, Phalan FC, Barter JW, et al. Axons of retinal ganglion cells are insulted in the optic nerve early in DBA/2J glaucoma. J Cell Biol. (2007) 179:1523-37. doi: 10.1083/jcb.200706181

49. Egg R, Reindl M, Deisenhammer F, Linington C, Berger T. Anti-MOG and anti-MBP antibody subclasses in multiple sclerosis. Mult Scler. (2001) 7:285-9. doi: 10.1177/135245850100700503

50. Wingerchuk DM, Lennon VA, Lucchinetti CF, Pittock SJ, Weinshenker BG. The spectrum of neuromyelitis optica. Lancet Neurol. (2007) 6:805-15. doi: 10.1016/S1474-4422(07)70216-8

51. Vargas ME, Watanabe J, Singh SJ, Robinson WH, Barres BA. Endogenous antibodies promote rapid myelin clearance and effective axon regeneration after nerve injury. Proc Natl Acad Sci USA. (2010) 107:11993-8. doi: 10.1073/pnas.1001948107

52. European Glaucoma Society Terminology and Guidelines for Glaucoma, 4th Edition-Chapter 2: classification and terminologySupported by the EGS Foundation: part 1: foreword; introduction; glossary; chapter 2 classification and terminology. Br J Ophthalmol. (2017) 101:73-127. doi: 10.1136/bjophthalmol-2016-EGSguideline.002

53. Zhou Y, Zhou B, Pache L, Chang M, Khodabakhshi AH, Tanaseichuk O, et al. Metascape provides a biologist-oriented resource for the analysis of systemslevel datasets. Nat Commun. (2019) 10:1523. doi: 10.1038/s41467-019-09234-6

Conflict of Interest: The authors declare that the research was conducted in the absence of any commercial or financial relationships that could be construed as a potential conflict of interest.

Copyright (c) 2021 Beutgen, Pfeiffer and Grus. This is an open-access article distributed under the terms of the Creative Commons Attribution License (CC BY). The use, distribution or reproduction in other forums is permitted, provided the original author(s) and the copyright owner(s) are credited and that the original publication in this journal is cited, in accordance with accepted academic practice. No use, distribution or reproduction is permitted which does not comply with these terms. 